\title{
Camera position adaptive compensation in IR sight line alteration
}

\author{
Jin-Song WANG ${ }^{1, a}$, Ji-Ming ZHANG ${ }^{1, b^{*}}$ and Yan-Feng $\mathrm{LI}^{1, \mathrm{c}}$ \\ ${ }^{1}$ Changchun University of Science and Technology,Jilin,Changchun 130022, China \\ asoldier_1973@163.com, b378874305@qq.com, ‘yannianyishou@126.com
}

Keywords: camera position;sight line alteration;adaptive compensation;infrared sights;

Abstract. In order to improve the measurement precision of infrared sight line alteration, a new adaptive correction algorithm of CCD camera pose small angle changing was proposed.Through the theoretical analysis, a mathematical model of the CCD camera pose calculation was established and a camera pose calculation formula was deduced, which was firstly applied to infrared sights zero momentum measurement.According to the infrared sight line alteration detection system,specific to the three kinds of camera pose, a contrast experiment that aiming sight line alteration coordinate was measured in the reference coordinate system was completed.Results showed that the calculating precision is 0.01 mil or less when camera did not tilt,and camera pose adaptive correction algorithm can effectively avoid the error of the infrared sight line alteration measurement caused by camera tilt, which provided a new method of camera position adaptive compensation for enhancing sight line alteration measurement precision.

\section{Introduction}

With the rapid development of infrared technology,infrared aiming is getting more and more attention.In weapon firing process, due to the objective lens sights, reticle,guide rails and other parts loosening,deformation or dramatic changes in ambient temperature can lead to changes in sight line alteration,which can affect the accuracy of the weapon.Domestic arsenal,research institutes commonly use conventional sight line alteration instrument,it consumes time, and has low digital level,it can not meet the current demand for infrared sights high efficiency and intelligent detection.In recent years, with the rapid development of photovoltaic technology and digital image processing technology. Although there are ways[1-6]to use CCD technology acquisition and digital interpretation for testing, but the measurement process, because the camera tilt detection accuracy is not high and the problem has yet to be solved.Reports rarely seen at home and abroad.

Based on machine vision technology, we ues image interpretation methods on the infrared sight line alteration measurement.We design a method of the camera posture adaptive compensation, which can effectively suppress the camera tilt measurement errors, and further enhance infrared sights walking zero measurement accuracy.

\section{System components and sight line alteration testing system method}

Sight line alteration testing system of the IR aiming sight consists of a reflective collimator, CCD camera with its installed components and a computer image interpretation sub-system. The reflective collimator includes a blackbody,targets, an off-axis paraboloid mirror and a plane mirror.As shown in Figure 1. 


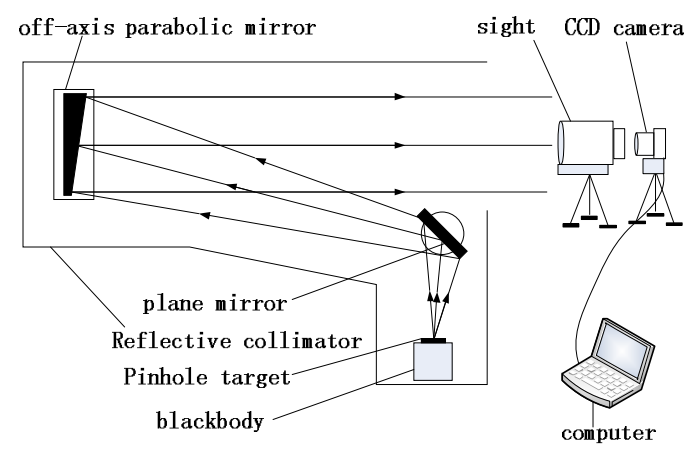

Fig.1 System composition diagram

Fix the measured IR aiming sight on sight mounts, and align the main optical axis of the infrared collimator.We can observe the target and aiming partition in the eyepiece field of view.CCD camera lens is placed in the exit pupil position of measured IR aiming sight.Adjust the eyepiece diopter of the measured IR aiming sight to zero diopter.By adjusting the CCD camera lens aperture and focal length properly,we can collect the clear images of target and sight reticle.Based on the collected images, the computer image interpretation sub-system can record the first interpretation of the sight reticle position $\mathrm{S}(\mathrm{xs}, \mathrm{ys})$ as zero position.Remove the sight.After a series of tests like firing, vibration, and temperature testing,install the sight again. Then we measure the reticle position $\mathrm{T}(\mathrm{xt}$, yt), and substitute it into the sight line alteration testing formulas (1) and (2)

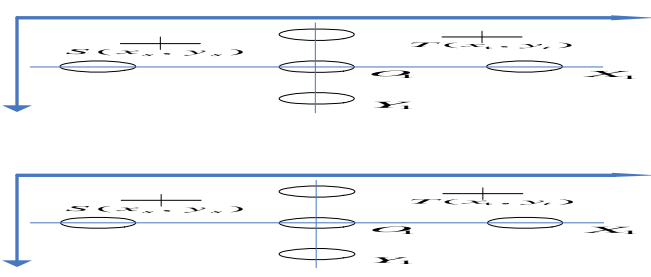

We can calculate the sight line alteration of direction XandY.Among them, $\theta_{\mathrm{x}}$ and $\theta_{\mathrm{y}}$ are the sight line alteration of direction $X$ and $Y$, the focal length of the collimator $f_{p}$ equals $1500 \mathrm{~mm}$.

\section{Camera position change on measuring precision}

The ideal sight line alteration testing method is:as shown in Figure 2,after installing the sight twice, we can get the aiming partition coordinate $S\left(x_{s}, y_{s}\right)$ and $T\left(x_{t}, y_{t}\right)$ in the coordinate system $\mathrm{X}_{1} \mathrm{O}_{1} \mathrm{Y}_{1}$, then we can get the sight line alteration by substituting them into the formulas (1) and (2).

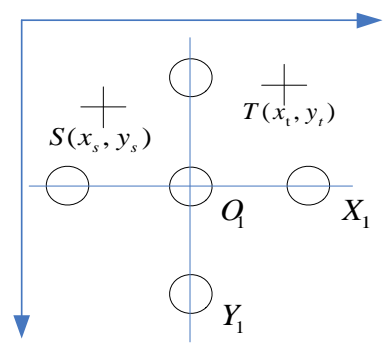

Fig.2 Sight baseline aiming variation schematic diagram

However,in the actual measurement,due to the vibration of measurement environment and carrying measured instruments because of site replacement can lead to small changes in camera pose.In addition, in the process of measurement the measured sight need to be repeated installed.In summary,these factors make the camera tilt and it is difficult to avoid.If the camera is tilted,the measurement will introduce errors.Figure 3 is a schematic diagram before and after the camera tilt. 


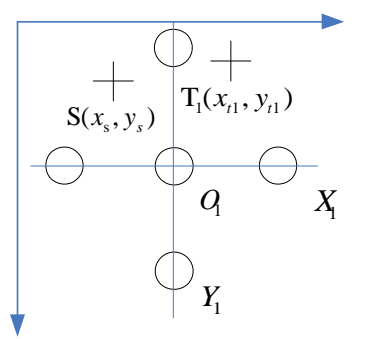

(a)CCD camera pose without tilt

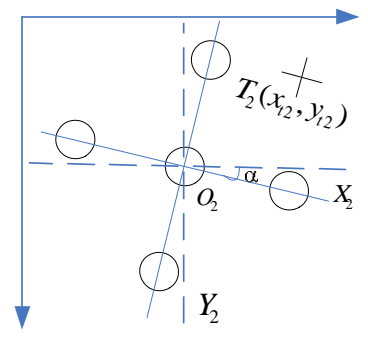

(b)CCD camera pose tilting

Fig. 3 The comparison of CCD camera pose change diagram

When the camera is not tilted,as shown in Figure3 (a).After the sight first installed, the coordinate of the aiming partition center in the coordinate system $\mathrm{X}_{1} \mathrm{O}_{1} \mathrm{Y}_{1}$ is $\mathrm{S}(-5.2,6.7)$. After the sight second installed, the coordinates of the aiming partition center is $\mathrm{T}_{1}(4.5,8.2)$. Then we can get the sight line alteration $\theta_{\mathrm{x} 1}$ and $\theta_{\mathrm{y} 1}$.

$$
\begin{gathered}
\theta_{x 1}=\left|\arctan \left(\frac{x_{s}}{f_{p}}\right)-\arctan \left(\frac{x_{t 1}}{f_{p}}\right)\right|=6.77 \mathrm{mil} \\
\theta_{y 1}=\left|\arctan \left(\frac{y_{s}}{f_{p}}\right)-\arctan \left(\frac{y_{t 1}}{f_{p}}\right)\right|=1.04 \mathrm{mil}
\end{gathered}
$$

When the camera is tilted at a small angle,as shown in Figure3 (b), the coordinate system $\mathrm{X}_{1} \mathrm{O}_{1} \mathrm{Y}_{1}$ turns to coordinate system $\mathrm{X}_{2} \mathrm{O}_{2} \mathrm{Y}_{2}$.If we don't correct the camera pose, we can cac ulate the coordinate of the aiming partition center $\mathrm{T}_{2}(5.9,7.1)$ in the reference coordinate sys tem $\mathrm{X}_{1} \mathrm{O}_{1} \mathrm{Y}_{1}$,which is shown in Figure3 (a).By substituting them into the formulas (1) and (2), we can get the sight line alteration $\theta_{\mathrm{x} 2}$ and $\theta_{\mathrm{y} 2}$.

$$
\begin{gathered}
\theta_{x 2}=\left|\arctan \left(\frac{x_{s}}{f_{p}}\right)-\arctan \left(\frac{x_{t 2}}{f_{p}}\right)\right|=7.68 m i l \\
\theta_{y 2}=\left|\arctan \left(\frac{y_{s}}{f_{p}}\right)-\arctan \left(\frac{y_{t 2}}{f_{p}}\right)\right|=0.28 \mathrm{mil}
\end{gathered}
$$

By the formulas(3)-(6), we can conclude when the camera pose without tilt, the sight line alteration of direction $\mathrm{X}$ is $6.77 \mathrm{mil}$ and the $\mathrm{Y}$ direction is $1.04 \mathrm{mil}$, while camera pose tilting,the sight line alteration of direction $\mathrm{X}$ is $7.68 \mathrm{mil}$ and the $\mathrm{Y}$ direction is $0.28 \mathrm{mil}$.Obviously, the measurement data vary greatly, and it introduces errors.

\section{The realization of the camera position adaptive compensation method}

The camera coordinate system treats the optical center of the camera as an origin,the optical axis of the camera as a Z-axis,image $\mathrm{X}, \mathrm{Y}$ axis as $\mathrm{X}$, Y-axis.If the camera is tilted,the camera coordinate deflects, but it donsen't change in the actual coordinate system. We need transform the camera coordinate system into the actual coordinate system.Suppose there is a point $\mathrm{P}$ in the space.Its homogeneous coordinates is $\left(\mathrm{x}_{\mathrm{w}}, \mathrm{y}_{\mathrm{w}}, \mathrm{z}_{\mathrm{w}}, 1\right)^{\mathrm{T}}$ in the actual coordinate system, and is $\left(\mathrm{x}_{\mathrm{c}}, \mathrm{y}_{\mathrm{c}}, \mathrm{z}_{\mathrm{c}}, 1\right)^{\mathrm{T}}$ in the camera coordinate system. We can achieve transformation between the camera coordinate system and the actual coordinates by formula(7). 


$$
\left[\begin{array}{c}
X_{C} \\
Y_{C} \\
Z_{C} \\
1
\end{array}\right]=\left[\begin{array}{cc}
R & T \\
0^{T} & 1
\end{array}\right] \times\left[\begin{array}{c}
X_{W} \\
Y_{W} \\
Z_{W} \\
1
\end{array}\right]=M_{1} \times\left[\begin{array}{c}
X_{W} \\
Y_{W} \\
Z_{W} \\
1
\end{array}\right]
$$

In which, $\mathrm{R}$ is a $3 \times 3$ orthogonal unit matrix, $\mathrm{T}$ is a three-dimensional translation vector, $0^{\mathrm{T}}=(0,0,0)$ ${ }^{\mathrm{T}}, \mathrm{M}_{1}$ is the connection matrix between the two coordinate systems.

In formation process of the infrared image,due to the blackbody temperature accuracy,distortion of the collimation system mirrors,inhomogeneity of the infrared radiation source and other factors' impact $^{[1]}$,it is easy to make the circular spot shape and size changes, and it is difficult to ensure that the coordinate system which is composed of five circular spot is a standard coordinate.

The camera pose adaptive compensation method designed in this paper,can not only transform the camera coordinate system into the actual coordinate system, but also reduce the coordinate system composed of five circular spot into a standard coordinate system. The specific algorithm is as follows:By gravity method ${ }^{[2-5]} \mathrm{we}$ can obtain five circular spot center coordinates $\mathrm{A}\left(\mathrm{x}_{\mathrm{A}}, \mathrm{y}_{\mathrm{A}}\right), \mathrm{B}\left(\mathrm{x}_{\mathrm{B}}, \mathrm{y}_{\mathrm{B}}\right), \mathrm{C}\left(\mathrm{x}_{\mathrm{C}}, \mathrm{y}_{\mathrm{C}}\right), \mathrm{D}\left(\mathrm{x}_{\mathrm{D}}, \mathrm{y}_{\mathrm{D}}\right)$ and $\mathrm{E}\left(\mathrm{x}_{\mathrm{E}}, \mathrm{y}_{\mathrm{E}}\right)$. With the horizontal axis DE, vertical axis $\mathrm{AB}$, we can establish a cartesian coordinate system,which is shown in Figure 4.

(A)

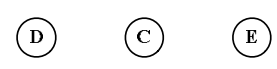

(B)

Fig.4 coordinate system determined by target points

Substitute $\mathrm{D}\left(\mathrm{x}_{\mathrm{D}}, \mathrm{y}_{\mathrm{D}}\right), \mathrm{E}\left(\mathrm{x}_{\mathrm{E}}, \mathrm{y}_{\mathrm{E}}\right)$ into the formula $(8)$.

$$
k_{1}=\frac{y_{E}-y_{D}}{x_{E}-x_{D}}
$$

We can obtain the slope of the horizontal axis DE is $\mathrm{k}_{1}$.that is the slope of the horizontal axis of the new coordinate system is $\mathrm{k}_{1}$. Rotate the horizontal axis $90^{\circ}$, We can obtain the longitudinal axis slope $\mathrm{k}_{2}$ by the formula(9).

$$
k_{2}=-\frac{1}{k_{1}}
$$

Substitute the A, B, C, D and E five target points' abscissas and the ordinates into equation (10) and (11),we can obtain targets center point coordinates $\mathrm{P}\left(\mathrm{x}_{0}, \mathrm{y}_{0}\right)$.

$$
\begin{aligned}
& x_{0}=\left(x_{A}+x_{B}+x_{C}+x_{D}+x_{E}\right) / 5 \\
& y_{0}=\left(y_{A}+y_{B}+y_{C}+y_{D}+y_{E}\right) / 5
\end{aligned}
$$

Using point to the straight line distance formula and target points' coordinates $\left(\mathrm{x}_{\mathrm{A}}, \mathrm{y}_{\mathrm{A}}\right), \mathrm{B}\left(\mathrm{x}_{\mathrm{B}}, \mathrm{y}_{\mathrm{B}}\right), \mathrm{D}\left(\mathrm{x}_{\mathrm{D}}, \mathrm{y}_{\mathrm{D}}\right), \mathrm{E}\left(\mathrm{x}_{\mathrm{E}}, \mathrm{y}_{\mathrm{E}}\right)$, we can obtain the distance's square of $\mathrm{A}, \mathrm{B}$ to the longitudinal axis $\Delta_{A Y}^{2}, \Delta^{2}{ }_{\mathrm{B} Y}$ and the distance's square of C,D to the longitudinal axis $\Delta^{2}{ }_{\mathrm{DX}}, \Delta^{2}{ }_{\mathrm{EX}}$. 


$$
\begin{aligned}
& \Delta^{2}{ }_{A Y}=\frac{\left(x_{A} * k_{2}-\left(1024-y_{A}\right)+y_{0}-k_{2} * x_{0}\right)^{2}}{1+k_{2}{ }^{2}} \\
& \Delta^{2}{ }_{\mathrm{BY}}=\frac{\left(x_{\mathrm{B}} * k_{2}-\left(1024-y_{\mathrm{B}}\right)+y_{0}-k_{2} * x_{0}\right)^{2}}{1+k_{2}{ }^{2}} \\
& \Delta_{\mathrm{D} X}^{2}=\frac{\left(x_{\mathrm{D}} * k_{1}-\left(1024-y_{\mathrm{D}}\right)+y_{0}-k_{1} * x_{0}\right)^{2}}{1+k_{1}{ }^{2}} \\
& \Delta_{\mathrm{EX}}{ }=\frac{\left(x_{\mathrm{E}} * k_{1}-\left(1024-y_{\mathrm{E}}\right)+y_{0}-k_{1} * x_{0}\right)^{2}}{1+k_{1}{ }^{2}}
\end{aligned}
$$

Combined formulas(12)-(15), we can get formula(16).

$$
\Delta_{\text {总 }}=\Delta_{\mathrm{AY}}^{2}+\Delta_{\mathrm{BY}}^{2}+\Delta_{\mathrm{DX}}^{2}+\Delta_{\mathrm{EX}}^{2}
$$

Estabilish the coordinate system by clockwise and counterclockwise rotation,as shown in Figure5.

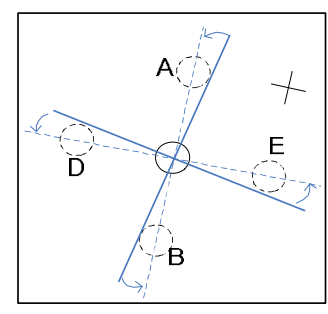

(a) anticlockwise rotation of coordinate axis

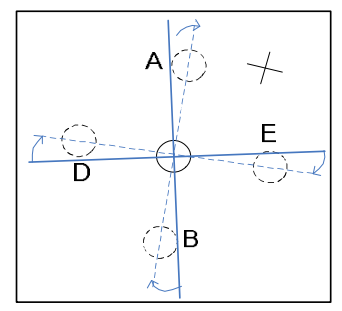

(b) clockwise rotation of coordinate axis

Fig.5 The correction of camera pose schematic diagram

Traversing the search area sequentially,we could calculate minimum distance's square $\Delta \mathrm{s}_{\min }$ and $\Delta^{\prime} \mathrm{S}_{\min }$ of counterclockwise and clockwise. The optimum slope $\mathrm{K}$ will be obtained by the formula (17).

$\mathrm{K}=\left\{\mathrm{K}_{\mathrm{i}} \mid \min \left\{\Delta \mathrm{s}_{\min }, \Delta^{\prime} \mathrm{s}_{\min }\right\}, \quad \mathrm{i}=1,2, \ldots, 400\right\}$

$\mathrm{i}=1,2, \ldots, 400$ defines a search range,it's an empirical value which is small enough for the angle of inclination. $\mathrm{K}$ is horizontal slope in the new coordinate system,we could calculate the slope of longitudinal axis-1 / $\mathrm{K}$ by formula(9). Then we can obtain transverse, longitudinal coordinates, and Aiming partition coordinatein the new coordinate system.Substituting them into formulas(1),(2), we could caculate the sight line alteration.

\section{Experiment and results analysis}

In order to verify the accuracy and stability of the method,we apply the method to the sight line alteration testing system of an IR aiming sight.

First,the CCD camera remains substantially parallel to the vertical plane.As shown in Figure 6 (b),rotate CCD camera clockwise about $9^{\circ}$.As shown in Figure 6 (c),rotate CCD camera anticlockwise about $11^{\circ}$. 


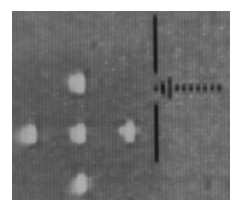

(a) camera without tilt

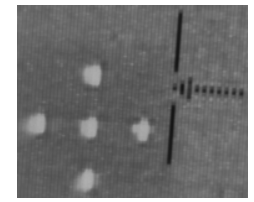

(b) camera rotated clockwise $9^{\circ}$

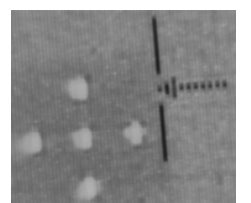

(c) camera rotated anticlockwise $11^{\circ}$

Fig.6 Three kinds of camera pose measured drawing

Then we apply the solution method proposed in this paper,In view of the three camera poses,we make contrast measuring of angular displacement which partition center to axis $\mathrm{X}$ and axis Y.The experimental data are shown in table 1.

Table1.Contrast experiment data table

\begin{tabular}{ccccccc}
\hline \multirow{2}{*}{$\begin{array}{c}\text { order } \\
\text { number }\end{array}$} & \multicolumn{3}{c}{ Zero momentum $\Theta_{\mathrm{x}}$ and $\Theta_{\mathrm{y}}$ when CCD camera is Tilted angle of $\alpha$.(mil) } \\
\cline { 2 - 7 } & $\Theta_{\mathrm{x} 1}$ & $\Theta_{\mathrm{y} 1}$ & $\Theta_{\mathrm{x} 2}$ & $\Theta_{\mathrm{y} 2}$ & $\Theta_{\mathrm{x} 3}$ & $\Theta_{\mathrm{y} 3}$ \\
\hline 1 & 8.30 & 3.81 & 8.30 & 3.80 & 8.31 & 3.80 \\
2 & 8.31 & 3.82 & 8.31 & 3.81 & 8.30 & 3.81 \\
3 & 8.30 & 3.81 & 8.29 & 3.80 & 8.30 & 3.80 \\
4 & 8.32 & 3.81 & 8.30 & 3.79 & 8.29 & 3.82 \\
5 & 8.30 & 3.81 & 8.29 & 3.80 & 8.31 & 3.80 \\
6 & 8.31 & 3.80 & 8.30 & 3.81 & 8.31 & 3.81 \\
7 & 8.30 & 3.81 & 8.30 & 3.79 & 8.30 & 3.80 \\
8 & 8.31 & 3.81 & 8.30 & 3.81 & 8.31 & 3.82 \\
9 & 8.30 & 3.80 & 8.29 & 3.80 & 8.30 & 3.81 \\
\hline standard deviation & 0.0071 & 0.0063 & 0.0074 & 0.0082 & 0.007 & 0.0082 \\
\hline average value & 8.30 & 3.80 & 8.31 & 3.79 & 8.31 & 3.80 \\
\hline
\end{tabular}

From Table1. When CCD camera without tilt,CCD camera rotated clockwise $9^{\circ}$ and CCD camera rotated anticlockwise $11^{\circ}$, the maximum deviation of $\theta_{x}$ is $0.006 \mathrm{mil}$, the minimum deviation of $\theta_{x}$ is $0.001 \mathrm{mil}$, and the maximum deviation of $\theta_{y}$ is $0.008 \mathrm{mil}$, the minimum deviation of $\theta_{y}$ is 0.001 mil.From the measuring data,the standard deviation $\sigma \leqslant 0.0082$, the repeatability is good.Therefore,whether the camera tilt or not,the application of this method can effectively suppress importing error,which can improve the measurement accuracy.

\section{Conclusion}

This paper designs a new method of camera pose adaptive compensation, which uses the adaptive compensation instead coordinate transformation, and establishes the camera pose calculation model.It's the first time to apply it to IR sight line alteration testing. The experimental results show that the method is low in cost,high degree of digitalization,easy operation,fast and effective.Besides the measurement accuracy is better than 0.01 mil. The method solve the problem that measurement errors which is caused by the camera tilt. 


\section{References}

[1] XIA QING,HU ZHENQI,WEI BEILEI, ETAL.New edge detection method for images of infrared thermal imager[J].Infrared and Laser Engineering, 2014,43(1):318-322(in Chinese)

[2] SU X ZH,JI H B,GAO X B. Detection method for dim small IR targets based on mathematical morphology[J].Infrared and Laser Engineer,2004,6(3):307-310.(in Chinese)

[3] CAI Wei-ke, Wen Gong-jian,Xi Fei,etal. A Method for Pose Estimation Based on Single Camera and Object Model[J].Journal of Image and Graphics,2008, 13(3):513-518(in Chinese)

[4] CAO Yu, Feng Ying,Zhao Li-shuang.Effect of attitude installation error of camera on precision of monocular visual odometry[J].Transducer and Microsystem Technologies,2012,31(12):23-30 (in Chinese)

[5] SINGH A,PETTERSSON R L,KARLHOLM J M.A two-stage approach for target identification and pose estimation ininfrared images[C].SPIE,2004,5613:177-188. 